\title{
Melhores práticas de enfermagem em educação em diabetes à criança hospitalizada: uma revisão integrativa
}

\author{
Luíza de Oliveira Messias Ortiz ${ }^{1}$, Elaine Buchhorn Cintra Damião², \\ Lisabelle Mariano Rossato ${ }^{3}$, Roberta Cristiane Pascarelli Alves ${ }^{4}$
}

\author{
${ }^{1}$ Enfermeira. Residente do Programa \\ Residência em Enfermagem na Saúde da \\ Criança e do Adolescente da Escola de \\ Enfermagem da Universidade de São Paulo / \\ Hospital Universitário da Universidade de São \\ Paulo. São Paulo, SP, Brasil. E-mail: \\ lu.messiasortiz@gmail.com. \\ 2 Enfermeira, Doutora em Enfermagem. \\ Professor Doutor MS-3.2 da Escola de \\ Enfermagem da Universidade de São Paulo. \\ São Paulo, SP, Brasil. E-mail: \\ buchhorn@usp.br. \\ ${ }^{3}$ Enfermeira, Doutora em Enfermagem. \\ Professor Doutor 2 da Escola de Enfermagem \\ da Universidade de São Paulo. São Paulo, SP, \\ Brasil. E-mail: rossato@usp.br. \\ ${ }^{4}$ Enfermeira, Mestre em Enfermagem. \\ Enfermeira da Unidade de Internação \\ Pediátrica do Hospital Universitário da \\ Universidade de São Paulo. São Paulo, SP, \\ Brasil. E-mail: \\ robertapascarelli@yahoo.com.br.
}

Recebido: 28/02/2017

Aceito: 23/10/2017.

Publicado: 31/12/2017.

Como citar esse artigo: Ortiz LMO, Damião EBC, Rossato LM, Alves RCP. Melhores práticas de enfermagem em educação em diabetes à criança hospitalizada: uma revisão integrativa. Rev. Eletr. Enf. [Internet]. 2017 [acesso em:

____19:a56. Disponível em:

http://doi.org/10.5216/ree.v19.45655.

\begin{abstract}
RESUMO
A incidência do Diabetes Mellitus tipo 1 (DM1) tem aumentado nos últimos anos, com consequente incremento de hospitalizações de crianças por primodescompensação diabética, com crescente necessidade de processo educativo. Diante desta realidade, objetivouse identificar na literatura as melhores práticas de enfermagem em educação em diabetes para crianças com DM1 hospitalizadas e sua família. Foi realizada uma revisão integrativa, com os descritores: Diabetic Ketoacidosis, Diabetes Education, Nursing e Child, Hospitalized, e busca livre em revistas de referência e artigos similares. Foram selecionados quatro estudos, e identificadas três categorias: Envolvimento e Empoderamento da Família no Processo Educativo em Diabetes; Atuação da Equipe Multiprofissional; Definição e Conteúdo do Processo Educativo, concluindo que o processo educativo deve incluir a família, ser realizado por uma equipe multiprofissional e fundamentado em evidências científicas. Poucos estudos foram encontrados, evidenciando necessidade de mais pesquisas na área. Descritores: Criança Hospitalizada; Educação em Saúde; Diabetes Tipo 1; Enfermagem Pediátrica.
\end{abstract}

\section{INTRODUÇÃO}

O Diabetes Mellitus tipo 1 (DM1) é uma doença autoimune crônica, causada pela destruição parcial ou total das células beta-pancreáticas das ilhotas de Langerhans, resultando na incapacidade progressiva de produzir insulina necessária ao metabolismo ${ }^{(1-2)}$. Possui diversos fatores genéticos predisponentes e alguns fatores ambientais pouco esclarecidos ${ }^{(1,3)}$, sendo responsável por 5-10\% dos casos totais de diabetes ${ }^{(1-2)}$.

O diabetes é a segunda doença crônica mais frequente da infância(4) e sua incidência está aumentando 
mundialmente nas últimas décadas ${ }^{(3)}$, em uma porcentagem de $3 \%$ ao ano, principalmente nas faixas etárias mais jovens ${ }^{(1,5-10)}$. Além disso, ressalta-se que $75 \%$ de todos os casos de DM1 são diagnosticados em indivíduos menores de 18 anos $^{(11-13)}$.

No Brasil, o DM1 ocorre em oito para cada 100.000 indivíduos com menos de 20 anos $^{(14)}$. Desde o diagnóstico é iniciado o uso de insulina, objetivando manter as glicemias ao longo do dia entre os limites de normalidade, evitando ampla variabilidade glicêmica. 0 tratamento também inclui o controle diário da glicemia capilar, dieta saudável, atividade física regular e o programa educacional. O tratamento visa não apenas o bom controle da doença, mas possibilitar crescimento e desenvolvimento adequados da criança $^{(2,10,15)}$.

Sabe-se que a maior parte das complicações agudas e crônicas do paciente com diabetes é devida ao mau controle metabólico ${ }^{(16-17)}$, sendo que seu manejo efetivo é um desafio ${ }^{(16,18)}$. A literatura evidencia a necessidade da educação em diabetes ${ }^{(16,18)}$, definida como processo de desenvolvimento de habilidades e incorporação de ferramentas imprescindíveis para atingir as metas do tratamento do diabetes. A educação é considerada a chave do sucesso do manejo da doença e essencial para a prevenção das complicações agudas e redução das complicações a longo prazo, devendo ser um processo contínuo, iniciado a partir do momento do diagnóstico(2,10-11,15,19-20).

Considerando a importância da educação em diabetes no manejo diário do tratamento, a fim de se evitar as complicações agudas e crônicas do diabetes na vida da criança, é mandatório que a equipe de saúde e em especial o enfermeiro, esteja capacitado para auxiliar a criança e sua família a adquirir o conhecimento e as habilidades necessárias ao efetivo manejo da situação de doença ${ }^{(15,20)}$.

Portanto, o presente estudo objetiva identificar na literatura quais são as melhores práticas na educação em diabetes para a criança com DM1 hospitalizada e sua família realizadas pelo enfermeiro.

\section{MÉTODO}

O estudo é uma revisão integrativa da literatura. Foram seguidas as subsequentes etapas: seleção da pergunta de estudo, estabelecimento de critérios para seleção da amostra, busca na literatura, definição de informações a serem extraídas dos estudos a serem selecionados, avaliação dos estudos incluídos, interpretação de resultados e apresentação da revisão(21).

A questão norteadora do estudo foi "Quais são as melhores práticas de educação em diabetes para a criança hospitalizada com DM1 utilizadas pelo enfermeiro descritas na literatura científica?". Utilizou-se, ainda, a estratégia PICO, onde:

- $\quad \mathrm{P}($ paciente $)=$ crianças/adolescentes hospitalizados por DM1;

- I (Intervenção) = melhores práticas de educação em diabetes;

- $\quad$ C (comparação) = não foi utilizada;

- $O$ (outcomes - desfecho) $=$ melhor controle e manejo da situação de doença.

Desse modo, foi possível identificar os descritores para a busca nas bases de dados: diabetic 
ketoacidosis, diabetes education, nursing e child, hospitalized, utilizando-se os operadores booleanos and, or e not em variadas combinações ${ }^{(22)}$.

A busca foi realizada nas bases de dados: Literatura Latino-Americana e do Caribe em Ciências da Saúde (LILACS), Scientific Eletronic Library Online (SciELO), Medical Literature Analysis and Retrieval System Online (PubMed) e Cummulative Index to Nursing and Allied Health Literature (CINAHL). Também foi realizada busca livre por meio de artigos similares aos selecionados nas bases de dados citadas e nas revistas internacionais cujo escopo é específico em diabetes, como The Diabetes Educator, Diabetes Care e Diabetes (Sociedade Brasileira de Diabetes).

Os critérios de inclusão para seleção das publicações foram os estudos primários publicados nos últimos 10 anos, de janeiro de 2007 a janeiro de 2017, devido ao pequeno número de publicações em menor período; nos idiomas inglês, espanhol e português e estudos realizados com crianças de zero a 18 anos incompletos submetidas à internação hospitalar. Foram excluídos capítulos de livros, teses de doutorado, dissertações de mestrado, relatos de caso e estudos relativos à educação ambulatorial e/ou em atenção primária à saúde.

O levantamento foi realizado nos meses de novembro/2016 a janeiro/2017, obtendo-se 122 estudos na base de dados PubMed, 701 na SciELO, 116 na CINAHL e nenhum na LILACS, além de 37 estudos encontrados por meio da busca livre, totalizando 1.026 publicações. Foram excluídos por título 883 estudos, 51 excluídos por resumo, nove publicações duplicadas e quatro artigos excluídos por indisponibilidade de acesso. Nesta etapa, 80 publicações foram eleitas e delas realizou-se leitura integral. Após essa análise, 76 estudos foram excluídos, sendo que 33 foram excluídos por abordarem educação em diabetes fora do ambiente hospitalar, 28 por não abordarem educação em diabetes, oito por serem revisões bibliográficas e ainda duas por abrangerem população adulta. Foram selecionados, portanto, quatro estudos para análise.

A síntese final foi feita de forma descritiva, referindo-se aos objetivos, resultados e conclusões obtidos de cada um dos estudos. O processo de seleção das publicações encontra-se representado no Figura 1. 


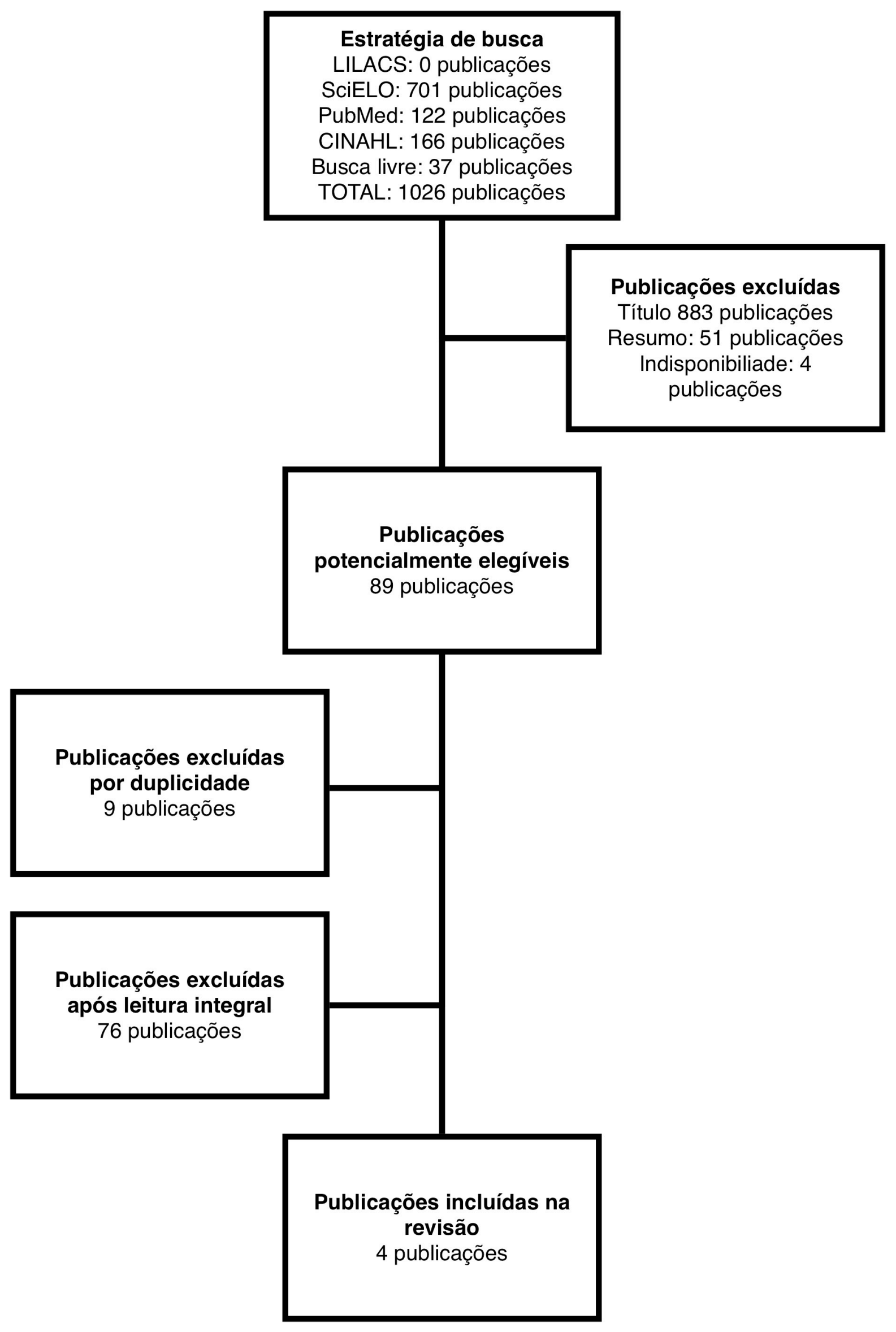

Figura 1: Estratégia de busca para seleção dos artigos incluídos para análise na revisão. 


\section{RESULTADOS}

Entre os quatro estudos selecionados para a análise, dois apresentavam abordagem qualitativa, um quantitativo (estudo pré e pós-intervenção) e um estudo retrospectivo. Apenas um estudo foi realizado no Brasil, em Fortaleza-CE, um foi realizado na Suécia e os demais nos EUA.

No Quadro 1 são apresentados os resultados e conclusões dos estudos incluídos nesta revisão.

Quadro 1: Resultados e conclusões dos estudos incluídos sobre educação em diabetes hospitalar para crianças e suas famílias.

\begin{tabular}{|c|c|c|c|}
\hline $\begin{array}{c}\text { Título/Pais de origem, ano } \\
\text { de publicação }\end{array}$ & Objetivos & Resultados & Conclusões \\
\hline $\begin{array}{l}\text { Pediatric Type } 1 \text { Diabetes: } \\
\text { Reducing Admission Rates for } \\
\text { Diabetes ketoacidosis(23) / } \\
\text { EUA, } 2016\end{array}$ & $\begin{array}{l}\text { Verificar se as admissões por } \\
\text { cetoacidose diabética (CAD), } \\
\text { indices de readmissões em } \\
\text { menos de } 30 \text { dias e tempo de } \\
\text { internação poderiam ser } \\
\text { reduzidos através de } 4 \\
\text { diferentes intervenções, } \\
\text { incluindo novo programa de } \\
\text { educação em diabetes. }\end{array}$ & $\begin{array}{c}\text { Redução estatisticamente } \\
\text { significativa nas } 3 \text { variáveis } \\
\text { de interesse. }\end{array}$ & $\begin{array}{l}\text { Admissões por CAD podem ser } \\
\text { reduzidas se aspectos } \\
\text { específicos do manejo do } \\
\text { diabetes, como por exemplo, a } \\
\text { educação aos pacientes e } \\
\text { familiares, forem atendidos. }\end{array}$ \\
\hline $\begin{array}{l}\text { Percepções de enfermeiras } \\
\text { acerca da prática educativa } \\
\text { no cuidado hospitalar a } \\
\text { crianças com diabetes }{ }^{(24)} \text { / } \\
\text { Brasil, } 2014\end{array}$ & $\begin{array}{l}\text { Descrição das percepções de } \\
\text { enfermeiras acerca da } \\
\text { educação em diabetes para } \\
\text { crianças internadas em } \\
\text { unidade hospitalar. }\end{array}$ & $\begin{array}{l}\text { Destaque para atuação da } \\
\text { enfermeira como } \\
\text { educadora; necessidade de } \\
\text { equipe multidisciplinar; } \\
\text { envolvimento da família; } \\
\text { importância da educação } \\
\text { no âmbito hospitalar; } \\
\text { estímulo à autonomia da } \\
\text { criança. }\end{array}$ & $\begin{array}{l}\text { Valorização de estratégias } \\
\text { educativas, intensificando } \\
\text { autonomia e autocuidado, } \\
\text { envolvendo a família. } \\
\text { Necessidade de } \\
\text { acompanhamento contínuo } \\
\text { por equipe multidisciplinar. }\end{array}$ \\
\hline $\begin{array}{l}\text { Long-Term Glycemic Control } \\
\text { as a Result of Initial } \\
\text { Education for Children With } \\
\text { New Onset Type } 1 \text { Diabetes - } \\
\text { Does the Setting Matter?(25) / } \\
\text { EUA, } 2013\end{array}$ & $\begin{array}{l}\text { Analisar a educação em } \\
\text { diabetes inicial dada em } \\
\text { centro medico acadêmico } \\
\text { (AMC) comparada à } \\
\text { educação em centros não } \\
\text { acadêmicos, no controle } \\
\text { glicêmico a longo prazo. }\end{array}$ & $\begin{array}{l}\text { Não houve diferença } \\
\text { significativa no controle } \\
\text { glicêmico a longo prazo } \\
\text { entre os dois grupos } \\
\text { comparados. }\end{array}$ & $\begin{array}{l}\text { O controle glicêmico a longo } \\
\text { prazo independe de uma } \\
\text { educação em diabetes feita em } \\
\text { centro especializado, sendo } \\
\text { mais importantes: reeducação } \\
\text { constante, foco nos estágios de } \\
\text { desenvolvimento infantil e } \\
\text { novos paradigmas } \\
\text { educacionais. }\end{array}$ \\
\hline $\begin{array}{l}\text { A multi-disciplinary education } \\
\text { process related to the } \\
\text { discharging of children from } \\
\text { hospital when the child has } \\
\text { been diagnosed with type } 1 \\
\text { diabetes - a qualitative } \\
\text { study }^{(26)} \text { / Suécia, } 2010\end{array}$ & $\begin{array}{l}\text { Entendimento do processo } \\
\text { de trabalho da equipe de } \\
\text { saúde especializada em } \\
\text { diabetes na educação de uma } \\
\text { criança recém diagnosticada } \\
\text { com DM1. }\end{array}$ & $\begin{array}{l}\text { A meta do programa de } \\
\text { educação em diabetes é } \\
\text { atingir um status de } \\
\text { autocuidado por parte do } \\
\text { paciente, sendo a família } \\
\text { parte integrante do } \\
\text { processo de cuidar. }\end{array}$ & $\begin{array}{l}\text { Recomendado programa de } \\
\text { educação em diabetes } \\
\text { desenvolvido especialmente } \\
\text { para cada família, usando } \\
\text { práticas clínicas recomendadas } \\
\text { por guidelines, incentivando a } \\
\text { família a fazer parte do } \\
\text { autocuidado da criança. }\end{array}$ \\
\hline
\end{tabular}

A análise dos objetivos, resultados e conclusões dos quatro estudos listados acima permitiu identificar três categorias: Envolvimento e Empoderamento da Família no Processo Educativo em Diabetes; Atuação da Equipe Multiprofissional; Definição e Conteúdo do Processo Educativo.

\section{Envolvimento e empoderamento da família no processo educativo em Diabetes}

Famílias revelam que o diagnóstico de DM1 da criança é uma situação devastadora e difícil de lidar 
inicialmente. A equipe de saúde deve ajudar a família a focar no processo educativo, tentando substituir a ansiedade e o estresse por assumir um papel ativo no cuidado da criança ${ }^{(26)}$. $O$ envolvimento da família na educação da criança é fundamental, da admissão à alta hospitalar, pois é a partir deles que a criança receberá ajuda para seu autocuidado ${ }^{(24)}$. O controle glicêmico da criança é diretamente relacionado à dinâmica familiar e pode ser afetado por problemas comportamentais ${ }^{(25)}$ e também pela variável socioeconômica ${ }^{(24)}$. Ressaltase que a criança aprecia o apoio recebido pela família em seu tratamento ${ }^{(24)}$.

O processo de educação em diabetes deve encorajar os pais e a criança a participar ativamente do seu autocuidado $^{(26)}$. A equipe deve compartilhar o cuidado da criança com a família ${ }^{(24)}$, estabelecendo uma parceria de sucesso na tríade profissionais-criança-família, desde o momento do diagnóstico da criança(25), auxiliando a família a incorporar as mudanças de modo efetivo ao seu estilo de vida ${ }^{(24)}$.

A criança deve ser estimulada a aplicar a própria insulina e realizar glicemia capilar, assumindo papel de protagonista do cuidado de acordo com seu desenvolvimento e capacidade ${ }^{(24)}$. A equipe deve promover na criança um sentimento de orgulho de seu autocuidado, tentando tornar o processo educativo divertido e interessante ${ }^{(26)}$.

O processo de prover a criança e família com conhecimentos e habilidades necessários para promover o autocuidado em diabetes, tomar decisões, resolver problemas e colaborar com a equipe de saúde é parte fundamental do processo de empoderamento do paciente ${ }^{(25)}$

\section{Atuação da equipe multiprofissional}

A enfermagem está mais próxima da criança e família desde a admissão hospitalar, sendo a grande responsável pela continuidade do cuidado em diabetes, especialmente o enfermeiro, pois consegue identificar as necessidades e preferências da família e a participação no cuidado da criança ${ }^{(24,26)}$. Sua atuaação é destacada como educadora na admissão hospitalar da criança com diabetes ${ }^{(24)}$. Mas a própria enfermagem reconhece a necessidade de uma equipe multiprofissional como estratégia de ensino e adesão ao tratamento, sendo imprescindível que este cuidado seja contínuo, não só no momento da admissão(24).

Os cuidados providos à criança pelos membros da equipe devem ser integrados, ocorrendo intersecção de conhecimentos e proporcionando qualidade e efetividade do processo educativo da criança, fornecendo apoio e informação uns aos outros ${ }^{(24,26)}$. Deve-se ter como prioridade as necessidades da criança de acordo com sua realidade e visão do mundo, e não focar apenas nas exigências terapêuticas ${ }^{(24)}$.

A equipe deve se manter atualizada quanto ao manejo do $D M 1^{(23)}$, e é desejável que o hospital possua um programa de educação em DM1 estruturado e padronizado ${ }^{(23)}$.

A construção de vínculo entre a equipe e a família é muito importante para o manejo do diabetes, sendo que um maior envolvimento da equipe impacta em melhor controle glicêmico a longo prazo(25).

\section{Definição e conteúdo do processo educativo}

O conceito de educação em DM1 é definido como processo para prover a criança e sua família com conhecimentos e habilidades essenciais para seu autocuidado, manejo de crises e realização de mudanças 
no estilo de vida ${ }^{(26)}$. A abordagem deve ser integral com ações que estimulem a participação da criança e sua família(24).

O processo iniciado desde a admissão hospitalar deve ser encarado como estratégia de apoio à criança e pautado no diálogo, com o propósito de aumentar o conhecimento sobre a doença e estimular o desejo do autocuidado pela criança e sua família(24,26). É importante lembrar que este processo deve ter continuidade após a alta hospitalar, e não deve ser restrito ao momento do diagnóstico, pelo contrário, será mantido por toda a vida da criança, requerendo contato frequente entre a tríade equipe-criança-família ${ }^{(25)}$.

O foco inicial, no momento do diagnóstico, é ensinar à criança e sua família as "ferramentas de sobrevivência" que permitirão que eles aprendam o suficiente para uma alta segura ${ }^{(25)}$. Portanto, deve-se ensinar aos membros da família: administração correta de insulina, monitoramento da glicemia capilar, ajustes alimentares e os cuidados com hipoglicemia ${ }^{(24,26)}$.

A internação hospitalar é usada para prover entendimento e habilidades necessários para a criança e família, sem que a expectativa da criança e sua família obtenham total entendimento sobre o $D M 1^{(26)}$. Vale lembrar que a experiência do diagnóstico de diabetes gera sofrimento para a criança e família, que pode se exacerbar com a internação hospitalar, gerando dúvidas e sobrecarga, podendo dificultar a compreensão das orientações realizadas e a aquisição das habilidades necessárias ${ }^{(24)}$. Além disso, é fundamental, que os conceitos sobre desenvolvimento infantil sejam integrados aos cuidados, traçando-se um plano educativo apropriado para cada criança ${ }^{(24)}$. Abaixo, segue o Quadro 2, resumindo tópicos a serem abordados na educação hospitalar da criança, de acordo com a fase de desenvolvimento, baseado em duas revisões de literatura ${ }^{(16,27)}$.

O fornecimento de material educativo didático, cultural e adequado à fase de desenvolvimento da criança é uma ferramenta importante do cuidado em diabetes, contando com uso de tecnologia, livros, panfletos e websites. Estes recursos promovem aumento da expressão verbal e favorecem o envolvimento e entendimento da criança sobre os cuidados com sua doença ${ }^{(23-24)}$.

A família deve ser convidada a fazer perguntas à equipe de saúde durante o processo educativo, e o enfermeiro deve revisar e reforçar o entendimento da mesma. Ele também deve testar se houve aquisição de conhecimento por parte da criança e família. É esperado que a família adquira habilidades para prover o cuidado à criança, como o manejo de caneta e/ou seringa de insulina, percepção do efeito da insulina e monitoramento dos níveis de glicemia capilar, dentre outros ${ }^{(26)}$.

Apesar das recomendações da $\operatorname{ADA}^{(20)}$ e $\operatorname{ISPAD}^{(2)}$ de que a educação em diabetes seja feita por uma equipe focada em diabetes pediátrico, o estudo realizado por Cabrera et al ${ }^{(25)}$ indica que não há evidências suportando que a educação inicial em diabetes deva ser realizada em centro especializado, visto que no estudo realizado não foi verificada diferença significativa nos níveis de hemoglobina glicada (HbA1c) apresentados no grupo pediátrico educado em unidade especializada em diabetes versus o grupo pediátrico educado em unidade não especializada ${ }^{(25)}$. 
Quadro 2: Tópicos essenciais para abordagem na educação da criança hospitalizada diagnosticada com diabetes e o quanto ela pode assumir seu autocuidado.

\begin{tabular}{|c|c|c|c|}
\hline $\begin{array}{c}\text { Fase de } \\
\text { desenvolvimento }\end{array}$ & $\begin{array}{c}\text { Características } \\
\text { relacionadas ao } \\
\text { diagnóstico de DM1 }\end{array}$ & Assuntos específicos para abordagem & $\begin{array}{l}\text { Empoderamento da criança e } \\
\text { autocontrole }\end{array}$ \\
\hline $\begin{array}{l}\text { TODDLER E PRÉ } \\
\text { ESCOLAR }\end{array}$ & $\begin{array}{l}\text { Alta dependência dos } \\
\text { pais e/ou cuidadores; } \\
\text { incapazes de detectar, } \\
\text { avaliar e verbalizar sinais } \\
\text { e sintomas do diabetes; } \\
\text { podem apresentar } \\
\text { manifestações clinicas } \\
\text { atípicas. }\end{array}$ & $\begin{array}{l}\text { Sinais de hipoglicemia e seus riscos } \\
\text { potenciais; Nutrição balanceada; Ajuste da } \\
\text { terapia insulínica e alimentação durante } \\
\text { períodos de infecção; Situações onde } \\
\text { criança rejeita a terapia; Sentimentos de } \\
\text { culpa; Integração social da criança; Suporte } \\
\text { social. }\end{array}$ & $\begin{array}{l}\text { Pais devem ter total controle da } \\
\text { administração de insulina, } \\
\text { monitoração dos níveis } \\
\text { glicêmicos e da alimentação da } \\
\text { criança até os } 3 \text { anos de idade. } \\
\text { Entre } 3 \text { e } 7 \text { anos, as crianças } \\
\text { podem começar a realizar } \\
\text { pequenas tarefas no seu } \\
\text { autocuidado ao diabetes. }\end{array}$ \\
\hline ESCOLAR & $\begin{array}{l}\text { Podem apresentar } \\
\text { quadros de depressão } \\
\text { moderada e ansiedade } \\
\text { ao diagnóstico. } \\
\text { Necessidade de } \\
\text { independência cada vez } \\
\text { maior. Compreendem } \\
\text { regras internas sobre } \\
\text { objetos e suas relações. }\end{array}$ & $\begin{array}{l}\text { Nutrição balanceada e o lidar com doces; } \\
\text { Adaptar a terapia insulínica no exercício } \\
\text { físico; Integração social na escola e } \\
\text { atividades de lazer; Segurança da criança } \\
\text { fora da supervisão dos pais; Suporte e } \\
\text { responsabilidade para a criança sem } \\
\text { excessos; Diabetes como instrumento de } \\
\text { poder; Importância do } \\
\text { automonitoramento glicêmico; } \\
\text { Comportamento típico ou problemas } \\
\text { emocionais da criança. }\end{array}$ & $\begin{array}{l}\text { Crianças podem assumir } \\
\text { progressivamente suas tarefas } \\
\text { diárias como aplicação de } \\
\text { insulina e monitoramento da } \\
\text { glicemia capilar, mas com a } \\
\text { supervisão de adultos } \\
\text { instruídos. } \\
\text { Pode ser instruída sobre a } \\
\text { fisiopatologia de sua doença de } \\
\text { forma simples. }\end{array}$ \\
\hline \multirow[b]{2}{*}{ ADOLESCENTES } & $\begin{array}{l}\text { Fase que compreende } \\
\text { diversas modificações } \\
\text { físicas, psíquicas e } \\
\text { sociais. }\end{array}$ & \multirow{2}{*}{$\begin{array}{l}\text { Dividir as responsabilidades do manejo do } \\
\text { diabetes entre a família; Promover } \\
\text { independência e autonomia; Influências da } \\
\text { puberdade no controle metabólico; Lidar } \\
\text { com mudanças de humor da idade; } \\
\text { Excesso de exercício físico e saídas } \\
\text { noturnas; Viagens sem os pais; Álcool e } \\
\text { drogas; Suporte motivacional; Sexualidade; } \\
\text { Transtornos alimentares; } \\
\text { Automonitoramento. }\end{array}$} & \multirow{2}{*}{$\begin{array}{l}\text { Deve ser responsável pelo seu } \\
\text { próprio tratamento, assumindo } \\
\text { o papel de protagonista. Porém } \\
\text { não significa que o cuidado e } \\
\text { responsabilidade sobre a doença } \\
\text { serão somente dele. Os pais } \\
\text { deverão estar presentes, } \\
\text { dividindo as tarefas e auxiliando } \\
\text { a promover autonomia. }\end{array}$} \\
\hline & $\begin{array}{l}\text { Já faz suas próprias } \\
\text { escolhas, a autonomia é } \\
\text { muito importante no } \\
\text { bom controle da doença. }\end{array}$ & & \\
\hline
\end{tabular}

\section{DISCUSSÃO}

Baseando-se nos resultados e conclusões do estudo, foram identificadas categorias importantes em relação à educação da criança diagnosticada com diabetes. Os três temas identificados são não excludentes, já que todos esses aspectos se entrelaçam no estabelecimento do processo educativo.

É bem esclarecido o conceito de educação em diabetes, enfatizado como estratégia de cuidado de abordagem integral da criança e família ${ }^{(24)}$. Percebe-se ser um processo contínuo que facilita o acesso ao conhecimento e promove o desenvolvimento de habilidades para o autocuidado e objetiva a melhoria na qualidade de vida(15-16).

A inclusão da família no cuidado, um dos grandes destaques do estudo, é corroborado pelas grandes instituições relacionadas a pesquisa em diabetes como a $A D A^{(20)}, \mathrm{SBD}^{(15)}$ e $\operatorname{ISPAD}^{(2)}$. Todos os guidelines apresentam a família como componente vital para o manejo excelente do diabetes durante a infância e adolescência $^{(20)}$.

A equipe de enfermagem deve estar atenta para fornecer uma educação compreensiva para a família 
e a criança ${ }^{(27)}$, atentando-se para potenciais limitadores do aprendizado, como por exemplo conflitos sociais, condições psiquiátricas, sobrecarga dos pais, problemas econômicos, falta de informação e algumas crenças espirituais ${ }^{(27-28)}$, além da hospitalização que é um fator de estresse adicional para a família(28-29).

A enfermagem deve constantemente avaliar a família submetida a seus cuidados ${ }^{(29-32)}$, pois a comunicação positiva e otimista entre a família relaciona-se a melhores índices glicêmicos e de adesão ao tratamento por parte da criança ${ }^{(32)}$, implicando em melhor qualidade de vida, ao passo que conflitos familiares, altos níveis de hostilidade e críticas, sentimento de falta de ajuda dos pais pela criança levam a resultados opostos ${ }^{(32)}$.

O enfermeiro deve incutir na família e na criança o desejo de aprender sobre a doença ${ }^{(27)}$ e a ter metas realistas para o alcance do autocontrole ${ }^{(27)}$, focando no alcance da independência e autonomia próprios de cada faixa etária(15-16). Uma participação ativa está diretamente ligada a um melhor controle glicêmico a longo prazo $^{(16,28)}$.

Outro ponto comum levantado por meio da análise é a participação não somente do enfermeiro na educação em diabetes, mas sim uma equipe multidisciplinar ${ }^{(2,15,20)}$. A participação de uma equipe multidisciplinar, que compartilhe da mesma filosofia e objetivos, tem efeitos benéficos metabólicos e psicológicos para as crianças durante o processo educativo.

Ressalta-se a necessidade de que a equipe esteja sempre atualizada quanto ao manejo do diabetes e que a instituição hospitalar possua um programa estruturado de educação em diabetes, guiado pelas diretrizes propostas pelas sociedades de especialistas ${ }^{(11,16,27)}$.

Encontra-se na literatura diversas fontes que indicam que o tratamento da criança diagnosticada com diabetes seja feito por uma equipe especializada nesta doença ${ }^{(11,16,27)}$, ou com pelo menos um membro especialista no assunto ${ }^{(31)}$, algo que Cabrera et $a^{(25)}$ contesta. Seu estudo mostrou que a educação da criança recém diagnosticada com diabetes feita em centro especializado para diabetes ou não, não interfere no controle glicêmico a longo prazo. Porém o próprio autor reconhece que há uma limitação neste estudo visto que a única variável analisada foi o nível de $\mathrm{HbA1}^{(25)}$. Sabe-se que os melhores resultados da educação em diabetes em crianças são mais impactantes na variável psicossocial do que no controle glicêmico ${ }^{(2,15)}$.

Esta análise encontrou evidências de que a educação em diabetes deve se iniciar assim que a criança é admitida no hospital, devendo ser continuada após a alta hospitalar, devido à importância de adaptar a terapia de acordo com as necessidades da criança e da família, tornando-se um processo contínuo ${ }^{(2,15,20,27,33)}$.

Não existe um modelo padrão mundial de educação à criança recém diagnosticada com diabetes, sendo que os métodos educativos podem variar mesmo sendo guiados por diretrizes consolidadas ${ }^{(16,28)}$. Este não é um problema, sendo inclusive necessária adaptação ao contexto da criança e informações individualizadas a cada família, que se adaptem à sua cultura e que sejam integrados à rotina de cuidados clínicos do hospital ${ }^{(2,15-16,18,20,27)}$.

O processo educativo para a criança no hospital deve ser baseado em metas ${ }^{(19)}$, com objetivos específicos, procurando focar em apenas uma meta terapêutica por vez, no lugar de focar no tratamento do 
diabetes como um todo, dada a sua alta complexidade ${ }^{(17)}$. Além disso, os cuidados não devem ser restritos somente à parte técnica, devendo incorporar as necessidades emocionais, sociais e psicológicas da criança e sua família ${ }^{(18)}$, além da rede externa de apoio que inspire confiança na criança ${ }^{(29)}$, o que auxilia na adesão ao tratamento.

O uso de tecnologias como celulares, jogos, torpedos, redes sociais, entre outras, são ferramentas educativas atrativas para as crianças e adolescentes, que se mostram promissoras nas intervenções, além de iniciativas criativas como bonecos e fantoches ${ }^{(15-16)}$.

A educação aos pais deve promover confiança e competência, e incluir um treinamento para aquisição de habilidades necessárias ${ }^{(27)}$. O uso de técnicas inovadoras como vinhetas e simulações com pacientes, que dão estrutura ao processo educativo sem acrescentar muita formalidade e permitem a interação dos pais no processo são extremamente benéficas ${ }^{(28)}$. Os materiais educativos fornecidos aos pais devem possuir linguagem apropriada e de fácil compreensão(2).

\section{CONCLUSÃO}

A enfermagem tem uma participação de destaque dentro da equipe multidisciplinar no processo educativo da criança com DM1 e sua família. Nesse sentido, o enfermeiro deve valorizar a parceria com a família no processo educativo, durante toda a hospitalização da criança, considerando que o envolvimento da família, desde a admissão até a alta da criança, seguindo por todo o seu desenvolvimento até a vida adulta, é essencial para um bom manejo do diabetes e contribui para o adequado controle glicêmico.

Destaca-se que a educação em diabetes durante a internação hospitalar deve ser parte do cuidado integral ao paciente, oferecido em meio a prática clínica, respeitando as individualidades e cultura da família, baseando-se em evidências científicas e de acordo com a fase de desenvolvimento e maturidade da criança.

Vale ressaltar que o momento do diagnóstico é uma fase de estresse para os pais e a criança, somado à própria hospitalização. Neste momento, a educação deve ser focada em "ferramentas de sobrevivência", promovendo uma alta hospitalar segura para a criança e assegurando a continuidade do cuidado.

Houve limitações neste estudo, visto que existem poucos estudos primários que abordem a educação em diabetes para crianças hospitalizadas. Considerando que a incidência do DM1 é crescente, pesquisas futuras sobre intervenções educativas são necessárias para assegurar o melhor cuidado para crianças diagnosticadas com DM1.

A contribuição desta revisão consiste em prover o enfermeiro com diretrizes para a prática educativa à criança hospitalizada com DM1, a fim de uniformizar condutas, garantir um processo educativo atualizado e que ofereça segurança e qualidade de vida à criança e sua família.

\section{REFERÊNCIAS}


1. Yogi-Morren D, Lansang MC. Management of Patients with Type 1 Diabetes in the Hospital. Curr Diab Rep [Internet]. 2014 [acesso em: 31 dez. 2017];14(2):458. Disponível em: http://doi.org/10.1007/s11892-013-0458-9. 2. International Society for Pediatric and Adolescent Diabetes. ISPAD Clinical Practice Consensus Guidelines 2014 [Internet]. Berlim: ISPAD; 2014 [acesso em: 31 dez. 2017]. Disponível em: https://www.ispad.org/?page=ISPADClinicalPract.

3. Negrato CA, Dias JPL, Teixeira MF, Dias A, Salgado MH, Lauris JR, et al. Temporal trends in incidence of Type 1 diabetes between 1986 and 2006 in Brazil. J Endocrinol Invest [Internet]. 2010 [acesso em: 31 dez. 2017];33(6):373-7. Disponível em: http://doi.org/10.1007/BF03346606.

4. Haller MJ, Atkinson MA, Schatz D. Type 1 Diabetes Mellitus: Etiology, Presentation, and Management. Pediatr Clin North Am [Internet]. 2005 [acesso em: 31 dez. 2017];52(6):1553-78. Disponível em:

http://doi.org/10.1016/j.pcl.2005.07.006.

5. Banion C, Valentine V. Type 1 diabetes throughout the life span. In: Mensing C, editor. The art and science of diabetes self-management education: a desk reference for healthcare professionals. Chicago: American Association of Diabetes Educators; 2006. p. 187-213.

6. Dabelea D, Mayer-Davis EJ, Saydah S, Imperatore G, Linder B, Divers J, et al. Prevalence of Type 1 and Type 2 Diabetes Among Children and Adolescents From 2001 to 2009. JAMA [Internet]. 2014 [acesso em: 31 dez.

2017];311(17):1778-86. Disponível em: http://doi.org/10.1001/jama.2014.3201.

7. Onkamo $P$, Väänänen $S$, Karvonen $M$, Tuomilehto J. Worldwide increase in incidence of Type I diabetes - the analysis of the data on published incidence trends. Diabetologia [Internet]. 1999 [acesso em: $31 \mathrm{dez}$.

2017];42(12):1395-403. Disponível em: http://doi.org/10.1007/s001250051309.

8. Patterson CC, Dahlquist GG, Gyürüs E, Green A, Soltész G. Incidence trends for childhood type 1 diabetes in Europe during 1989-2003 and predicted new cases 2005-20: a multicentre prospective registration study. Lancet [Internet]. 2009 [acesso em: 31 dez. 2017]s;373(9680):2027-33. Disponível em: http://doi.org/10.1016/S0140-6736(09)60568-7. 9. Gyurus EK, Patterson C, Soltesz G. Twenty-one years of prospective incidence of childhood type 1 diabetes in Hungary - the rising trend continues (or peaks and highlands?). Pediatr Diabetes [Internet]. 2012 [acesso em: 31 dez. 2017];13(1):21-5. Disponível em: http://doi.org/10.1111/j.1399-5448.2011.00826.x.

10. International Diabetes Federation. IDF Diabetes Atlas Seventh Edition [Internet]. 7th ed. Bruxelas: International Diabetes Federation; 2015. Disponível em:

https://www.idf.org/component/attachments/attachments.html?id=1093\&task=download.

11. Hermoso López F, Barrio Castellanos R, Garcia Cuartero B, Gómez Gila A, González Casado I, Oyarzabal Irigoyen M, et al. Asistencia al niño y adolescente con diabetes. Unidades de referencia en diabetes pediátrica. An Pediatría [Internet]. 2013 [acesso em: 31 dez. 2017];78(5):335.e1-335.e4. Disponível em:

http://doi.org/10.1016/i.anpedi.2012.10.001.

12. Office Guide to Diagnosis and Classification of Diabetes Mellitus and Other Categories of Glucose Intolerance. Diabetes Care [Internet]. 1995 [acesso em: 31 dez. 2017];18(Suplemento 1):3-4. Disponível em: http://doi.org/10.2337/diacare.18.1.S3.

13. Oram RA, Patel K, Hill A, Shields B, McDonald TJ, Jones A, et al. A Type 1 Diabetes Genetic Risk Score Can Aid Discrimination Between Type 1 and Type 2 Diabetes in Young Adults. Diabetes Care [Internet]. 2016 [acesso em: 31 dez. 2017];39(3):337-44. Disponível em: http://doi.org/10.2337/dc15-1111.

14. The DIAMOND Project Group. Incidence and trends of childhood Type 1 diabetes worldwide 1990-1999. Diabet Med [Internet]. 2006 [acesso em: 31 dez. 2017];23(8):857-66. Disponível em: http://doi.org/10.1111/j.1464-

5491.2006.01925.x.

15. Sociedade Brasileira de Diabetes. Diretrizes da Sociedade Brasileira de Diabetes 2015-2016 [Internet]. São Paulo: A. C. Farmacêutica; 2016 [acesso em: 31 dez. 2017]. Disponível em:

http://www.diabetes.org.br/profissionais/images/docs/DIRETRIZES-SBD-2015-2016.pdf.

16. Leite SAO, Zanim LM, Granzotto PCD, Heupa S, Lamounier RN. Pontos básicos de um programa de educação ao paciente com diabetes melito tipo 1. Arq Bras Endocrinol Metabol [Internet]. 2008 [acesso em: 31 dez. 2017];52(2):233-42. Disponível em: http://doi.org/10.1590/S0004-27302008000200010.

17. Coffen RD, Dahlquist LM. Magnitude of Type 1 Diabetes Self-management in Youth Health Care Needs Diabetes Educators. Diabetes Educ [Internet]. 2009 [acesso em: 31 dez. 2017];35(2):302-8. Disponível em:

http://doi.org/10.1177/0145721708327534. 
18. Chaney D. Structured diabetes education for children and adolescents. Nurs Stand [Internet]. 2012 [acesso em: 31 dez. 2017];27(6):41-7. Disponível em: https://doi.org/10.7748/ns2012.10.27.6.41.c9350.

19. Wang J, Siminerio LM. Educators' Insights in Using Chronicle Diabetes. Diabetes Educ [Internet]. 2013 [acesso em: 31 dez. 2017];39(2):248-54. Disponível em: http://doi.org/10.1177/0145721713475844.

20. Standards of Medical Care in Diabetes - 2017: Summary of Revisions. Diabetes Care [Internet]. 2017 [acesso em: 31 dez. 2017];40(Suplemento 1):S4-5. Disponível em: http://doi.org/10.2337/dc17-S003.

21. Mendes KDS, Silveira RCCP, Galvão CM. Revisão integrativa: método de pesquisa para a incorporação de evidências na saúde e na enfermagem. Texto Context - Enferm [Internet]. 2008 [acesso em: 31 dez. 2017];17(4):758-

64. Disponível em: http://doi.org/10.1590/S0104-07072008000400018.

22. Santos CMC, Pimenta CAM, Nobre MRC. The PICO strategy for the research question construction and evidence search. Rev Lat Am Enfermagem [Internet]. 2007 [acesso em: 31 dez. 2017];15(3):508-11. Disponível em:

http://doi.org/10.1590/S0104-11692007000300023.

23. Ilkowitz JT, Choi S, Rinke ML, Vandervoot K, Heptulla RA. Pediatric Type 1 Diabetes: Reducing Admission Rates for Diabetes Ketoacidosis. Qual Manag Health Care [Internet]. 2016 [acesso em: 31 dez. 2017];25(4):231-7. Disponível em: http://doi.org/10.1097/QMH.0000000000000109.

24. Pennafort VPS, Silva ANS, Queiroz MVO. The perception of nurses regarding educational practices for children with diabetes in hospital care. Rev Gaucha Enferm [Internet]. 2014 [acesso em: 31 dez. 2017];35(3):130-6. Disponível em: http://doi.org/10.1590/1983-1447.2014.03.43313.

25. Cabrera SM, Srivastava NT, Behzadi JM, Pottorff TM, Dimeglio LA, Walvoord EC. Long-term glycemic control as a result of initial education for children with new onset type 1 diabetes: does the setting matter? Diabetes Educ [Internet]. 2013 [acesso em: 31 dez. 2017];39(2):187-94. Disponível em: http://doi.org/10.1177/0145721713475845. 26. Jönsson L, Hallström I, Lundqvist A. A multi-disciplinary education process related to the discharging of children from hospital when the child has been diagnosed with type 1 diabetes--a qualitative study. BMC Pediatr [Internet]. 2010 [acesso em: 31 dez. 2017];10:36. Disponível em: http://doi.org/10.1186/1471-2431-10-36.

27. Lange K, Sassmann H, von Schütz W, Kordonouri O, Danne T. Prerequisites for age-appropriate education in type 1 diabetes: a model programme for paediatric diabetes education in Germany. Pediatr Diabetes [Internet]. 2007 [acesso em: 31 dez. 2017];8 Supl. 6:63-71. Disponível em: http://doi.org/10.1111/j.1399-5448.2007.00277.x.

28. Ramchandani N, Johnson K, Cullen K, Hamm T, Bisordi J, Sullivan-Bolyai S. CDE Perspectives of Providing NewOnset Type 1 Diabetes Education Using Formal Vignettes and Simulation. Diabetes Educ [Internet]. 2017 [acesso em: 31 dez. 2017];43(1):97-104. Disponível em: http://doi.org/10.1177/0145721716676893.

29. Nascimento LC, Amaral MJ, Sparapani VC, Fonseca LMM, Nunes MDR, Dupas G. Diabetes mellitus tipo 1: evidências da literatura para seu manejo adequado, na perspectiva de crianças. Rev Esc Enferm USP [Internet]. 2011 [acesso em: 31 dez. 2017];45(3):764-9. Disponível em: http://doi.org/10.1590/S0080-62342011000300031. 30. Edge JA, Ackland F, Payne S, McAulay A, Hind E, Burren C, et al. Care of children with diabetes as inpatients: frequency of admissions, clinical care and patient experience. Diabet Med [Internet]. 2013 [acesso em: 31 dez. 2017];30(3):363-9. Disponível em: http://doi.org/10.1111/dme.12059.

31. Edge J, Ackland FM, Payne S, McAuley A, Hind E, Burren C, et al. Inpatient care for children with diabetes: are standards being met? Arch Dis Child [Internet]. 2012 [acesso em: 31 dez. 2017];97(7):599-603. Disponível em: http://doi.org/10.1136/archdischild-2011-301212.

32. Ridge K, Thomas S, Jackson P, Pender S, Heller S, Treasure J, et al. Diabetes-Oriented Learning Family Intervention (DOLFIN): a feasibility study evaluating an intervention for carers of young persons with Type 1 diabetes. Diabet Med [Internet]. 2014 [acesso em: 31 dez. 2017];31(1):55-60. Disponível em: http://doi.org/10.1111/dme.12333.

33. Jaacks LM, Bell RA, Dabelea D, D’Agostino RB, Dolan LM, Imperatore G, et al. Diabetes Self-Management Education Patterns in a US Population-Based Cohort of Youth With Type 1 Diabetes. Diabetes Educ [Internet]. 2014 [acesso em: 31 dez. 2017];40(1):29-39. Disponível em: http://doi.org/10.1177/0145721713512156. 